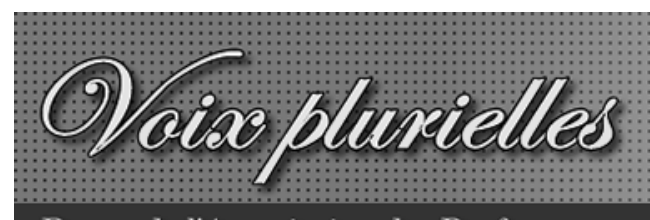

Revue de l'Association des Professeur-e-s de Français des Universités et Collèges Canadiens

\author{
Voix plurielles \\ Volume 4, Numéro 1 : mai 2007
}

Daniel Cohen

\title{
Liliane Atlan et les ambiguïtés d'une réception
}

Citation MLA : Cohen, Daniel. «Liliane Atlan et les ambiguïtés d'une réception .» Voix plurielles 4.1 (mai 2007).

(C) Voix plurielles, revue électronique de l'APFUCC 2007. 


\title{
Liliane Atlan et les ambiguïtés d'une réception
}

\author{
Daniel Cohen
}

Mai 2007

$\mathrm{L}$

e paradoxe, concernant Liliane Atlan, ne ressortit pas à l'existence de ses ouvrages mais à leur réception, et peut-être même davantage à leur diffusion.

Voici l'un des auteurs de langue française, parmi les plus originaux de ce dernier demisiècle, qui a su bouleverser et fidéliser un peu plus qu'une poignée d'admirateurs, mais qui n'a pu briser l'omerta des décideurs dont dépend une mutation cruciale : faire d'untel un écrivain que le grand public place dans la liste de ses repères culturels. Cependant le philosophe Gérard Israël, dans un dossier à elle consacré, voici une dizaine d'années, paru dans les Nouveaux Cahiers, baptisés depuis Les Cahiers du Judaïsme, avait eu raison de la considérer comme l'un des grands écrivains juifs de ce temps.

On pourrait disserter à l'infini sur la qualité « d'écrivain juif », en Occident notamment, tant les contradictions abondent et pourraient susciter, à bon droit, la discussion, voire la récusation.

Proust, Kafka, Harold Pinter, — et ainsi remonter de fil en aiguille jusqu'à Montaigne — tutti quanti, écrivains juifs ? Serait-ce la naissance qui générerait l'écriture, ou au contraire le milieu, les événements psychologiques, voire historiques qui la galvaniseraient à partir d'une complexion réfractaire à toute approche ethnique ? Or Liliane Atlan se reconnaît dans la qualification, la revendique et même explique le silence à l'entour de son travail de dramaturge. Il n'est pas, dans l'intention du signataire de cet article, de voir en quoi son soupçon recoupe une situation objective et un phénomène social incontesté. Son approche sera plus ample dans un ouvrage qu'il prépare sur cet auteur, qui devra tenir compte de l'ensemble de l'œuvre, puisque les inédits qui la composent sont, à ce jour, aussi importants que le corpus publié.

Par « silence », nous n'entendons pas vide complet. Il n'est pas d'année qui ne voit l'une ou l'autre des pièces d'Atlan montée à Paris et ailleurs et il n'en est pas qui passe sans qu'un article, de qualité fort inégale il est vrai, ne vienne rappeler, chacun à sa manière, la dette que nous avons contractée à l'égard d'un écrivain engagé, et si profondément, dans sa propre dette envers un passé proche dont elle n'a réchappé que par miracle.

Par « silence », nous visons l'absence de Monsieur Fugue, ou d'un Opéra pour Terezin, ou des Mers Rouges, dans le répertoire des grandes scènes françaises, et partant des scènes internationales. Dieu seul sait que ce théâtre, à la fois magnifique, épique et comique, jusque dans le tragique qui 
tisse sa trame, dispose de la fonction sacrée que toute œuvre dramatique majeure sécrète... Dieu seul sait, encore, par quelle splendeur poétique, les opus de Liliane Atlan ont su exhausser un substrat abstrait à l'extrême jusque dans les années $80:$ l'extermination des juifs européens par les peuples européens. Au siècle de fer.

NOUS voyons réunis, dans le paradoxe posé en exorde, une articulation insolite : un auteur, dont l'œuvre harmonieuse pose un défi, n'a pas pu renverser moins une conspiration du silence qu'un malaise général dû à la reconnaissance d'une vérité et à l'incapacité des décideurs de l'imposer avec éclat. Tel prestige, pour ne pas sombrer, est retenu par telle institution prestigieuse, le théâtre de France-Culture par exemple, comme si, pour se dédouaner d'y parvenir jamais, une société que la culture agrège, accorde l'une de ses lumières, en attendant que l'audace paie le jour venu.

Triste affaire...

Le précédent d'Imre Kertesz nous enseigne qu'il n'est d'œuvre d'exception qui ne s'affirme mutatis mutandis. Il a fallu des dizaines d'années d'attente à l'écrivain hongrois, prix Nobel de littérature, avant qu'il ne s'arrache à la misérable obscurité en laquelle l'avaient confiné un milieu peu soucieux de gratter ses effroyables compromissions. Autrefois, à l'heure du démon. Il est possible que nous citions cet exemple à titre de consolation, sinon d'exorcisme; nous n'ignorons pas la quantité de conservatismes dont la scène, voire la culture françaises sont lestées ces jours-ci.

Le «silence » que nous évoquions tantôt ressemble davantage à une paresse générale qu'à un grand trou noir : tout, dans cette œuvre, devrait concourir à l'allumage des lumières. Y aurait-il si peu de place, en Occident, au partage des émotions que ce théâtre qui, tout en ne s'assignant pas à la produire expressément, est un théâtre de l'émotion la plus haute ? Il ose, lui, affronter l'épouvante à travers mille mots simples et sobres. Ils disent, eux, notre humanité conquérante, soumise et humiliée, ou ambivalente...

Il est assez singulier que les pièces d'Atlan suscitent, à la fermeture du rideau, un silence recueilli, un bouleversement profond, mêlé de stupeur et de joie étrange, que vont bientôt rompre les acclamations, presque gênées : l'aventure, en commun, celle du texte, celle des acteurs, nous promène loin dans ce qu'il est convenu d'appeler l'indicible; une convention de ce type paraît déplacée, révèle le décalage entre un théâtre qui sait rendre la violence extrême des hommes, par la seule vertu de ses mots, et précisément le théâtral qui nous apparait désynchronisé. S'il y a eu subjugation, elle n'a émané que de l'art très tenu de l'auteur. Il s'est donc agi d'autre chose : on a communié dans un état spirituel au fur et à mesure que le récit tragique s'est décanté. Restauration in fine d'une part en nous déchirée qu'on a étrangement, à travers un cheminement intransigeant, apaisée fragment après fragment et à contre-courant de tout pathétique. Sanctuaire ? Celui d'une humanité revalorisée ? Intense INSTANT au cours duquel les spectateurs se sont moins délivrés du poids des morts, de leurs morts, que de la Mort, personnage qu'évoque Paul Celan dans un vers fameux adressé à l'Allemagne; je songe aussi au verset 10, 2 du Lévitique que Rachi commente ainsi : «... Qu'ils n'entrent pas enivrés dans le Sanctuaire ». Levinas se 1'est approprié en introduction à sa Difficile liberté.

S'il en fut qui vinrent dans les spectacles susdits, possédés de l'excitation attachée à l'idée de divertissement, fût-il pascalien, ce théâtre-là aura tôt fait de les aiguiller ailleurs : quand se jouent des rencontres insoupçonnées - a) entre part au destin terrestre et Soi ; b) part aux mythes 
universels dont la Mort est l'une des ponctuations. un !

LILIANE ATLAN inaugura son théâtre par Monsieur Fugue : pour un coup de maître, c'en fut

Depuis, cette pièce est un « classique » : ce n'est pas la plus forte de son répertoire, mais c'est devenu presque une légende. Vingt ans après qu'on eut éteint les fours à Auschwitz et ailleurs, dans la constellation de l'anéantissement des corps et de leurs pensées, on donnait en France, sur la scène et dans les librairies, un texte, paru alors aux éditions du Seuil, d'une rare élévation, précisément parce qu'il détruisait de l'intérieur l'idée quasi monolithique d'un ennemi qui aurait été criminel dans sa totalité ; l'arrachant à la dangereuse prévention qu'on pouvait tirer de cette entreprise, à la mesure même de son effroyable accomplissement, l'Allemagne était, à travers la figure de Fugue, un pays « trop humain », qui disposait de la capacité du remords et de la culpabilité ; le mouvement de 68 fit le reste : depuis, l'Allemagne réunifiée est la première des démocraties européennes. Une visite à Berlin suffit à démontrer un mea culpa maxima qu'aucun pays n'a su mener à ce rythme et avec cette sincérité des fils écœurés jetant à l'encan les lâchetés et les ivresses des pères.

Par un mécanisme intuitif propre aux grands écrivains, Liliane Atlan faisait jouer, peu de temps avant ladite révolution, ce qui avait jailli d'elle dans l'urgence : comme un corps qui s'extrait d'un corset qu'il ne supporte plus. À cette abscisse-là, par cette tension-là, et avec ce tempérament-là, il ne pouvait évidemment pas s'agir d'un manifeste mais, à sa manière et selon ses propres codes, d'une œuvre prophétique : l'Allemagne se réveillerait et observerait d'un œil neuf son ancien creuset.

Le tueur nazi en puissance, devenant accompagnateur de la mort des enfants, infuse dans son odyssée la part complexe d'une humanité étrange mais qui a l'avantage d'exister. Fugue, à son insu, porte dans ses veines le flux d'un repentir qui permettra, un jour, au peuple dont il se réclame et au nom duquel il se croit missionné pour tuer des enfants, de se regarder dans le miroir et découvrir sa tête de Janus.

La pièce fut applaudie à Saint-Étienne, à Paris, partout en Europe, en Israël. Elle a eu pour effet d'attirer les lumières sur la dramaturge et en même temps de les tamiser. Comprenant qu'elle avait une voie à suivre, quels qu'en fussent les écueils, refusant de se disperser dans les compromis d'une gloire qu'on lui marchanderait peut-être ou dans les ambiguïtés du prestige, Liliane Atlan préféra prendre vingt ans à sa vie afin de méditer et d'ériger des monuments qui oseraient se mesurer aux créations les plus complexes, ceux qu'on jouerait à travers le temps sans craindre le déphasage dû à toute modernité. Vieux débat : vous en trouverez l'écho à chacune des marches de l'histoire du théâtre en Occident.

Grâces soient donc rendues à notre écrivain de s'être tenu à l'écart d'un théâtre factuel avec ses ors et ses falbalas, articles que l'on accouple à « avant-garde » pour se donner du contant et du jusant... S'il y a eu désert, il en est peu qui sachent l'engendrer de ces constructions polyphoniques dont Un Opéra pour Terezin incarne à lui tout seul l'excellence. Les opus des années 90, théâtre à la fois très écrit et très neuf dans sa conception, ressemblent à des rituels religieux grandioses ; prenons garde d'employer le mot « religieux » sans précaution en une époque où il est convoqué comme geste d'affranchissement ou de revendication : ni Un opéra pour Terezin ni Les Mers Rouges ne ressortissent à la prière militante, et comment la contiendraient-ils tant leur charge en 
circonscrit, par essence, toute tentation ? S'il y a prière, c'est dans la nécessité collective d'établir, par la mémoire, un lien puissant entre les vivants, je préfèrerais même écrire les survivants, et ceux que l'on a condamnés à mort puis exécutés sans procès public, dans l'ombre, loin, si loin de leurs foyers en ces temps où les chefs d'une nation avancée, infatués de leurs bondieuseries, nous révélaient que la civilisation la plus raffinée est davantage productrice de barbarie que celle que l'on croit et prétend inférieure...

SON massif dramatique assure à Liliane Atlan une place à part dans le répertoire théâtral de langue française ; son thème ne lui est pas spécifique : il s'est éparpillé ailleurs, et franchement dans des œuvres disparates. Elle reste à ce jour le seul auteur qui soit aisément reconnaissable et dont le travail, parce qu'il puise dans la tradition, dispose d'une faculté à la pérennisation. C'est à la fois les conditions d'écriture et l'émotion que cette dernière contrôle et diffuse, avec une surprenante économie de moyens, qui l'assurent. On eût aimé que la pavane jeuniste qui foudroie la scène contemporaine fût un tout petit moins vorace et laissât à quelques auteurs considérables exprimer un peu de leur génie : face à la pesée d'un misérable magnificat que brodent pathos et subversion molle, ceux du verbe actuel, le style allusif d'Atlan, cette manière qu'elle a de faire coexister, d'un même trait, le rire, les larmes, le cosmos, semblent voguer à des années-lumière. Son théâtre serait-il fait pour un banquet des anges ? Je ne le crois pas. Les bacchanales d'une société de bas-empire l'indiffèrent mais ne l'indiffère pas le côté suiviste d'un système qui, par manque de courage, cèle d'avance toute loyale ouverture à un théâtre de la mémoire...

L'AUTRE travail, en prose et en poésie, n'est pas moins fort (le dernier recueil, Le Maître-Mur est sorti chez Dumerchez en 2004). Son autobiographie agrège quatre textes; un titre les réunit : Petites bibles pour mauvais temps; rarement on aura vu autobiographie plus allégorique, au style midrashique, composition fluide et ironique, propre à ces conteurs qui, à travers les siècles, se sont attaqués à la narration avec deux armes : la simplicité et la dérision.

Pour autant, Liliane Atlan n'est pas ébranchée de son temps : les techniques d'écriture qui lui sont propres l'aident à édifier ses projets et à les parachever. Il n'est pas d'ouvrage comparable au Rêve des Animaux rongeurs, monologue d'une narratrice qui halète quasiment devant une union naufragée : un sujet, banal entre tous, passe à l'état de fable, de métaphore, d'apologue ; c'est une transposition mystique, une méditation syncopée de sanglots : ceux du regret et de la liberté enfin reconquise. Dans la catégorie des textes monologués, je place Le Rêve des Animaux rongeurs aux côtés des plus grands dans le genre; c'est peut-être aussi le chef d'œuvre absolu de l'écrivain.

Or, diriez-vous, à quoi attribuer le silence qui enserre cette œuvre majestueuse, œuvre maudite s'il en est? Quel est donc le contresens qui la greffe : des titres d'une beauté rare ici - en face la pénombre ? Une réputation certaine d'un côté — les moyens ingrats mis à sa disposition pour briser le cercle vicieux ? Ces questions et d'autres prescriraient une approche plus affinée en ce qu'elles requerraient une étude éclairée de la sociologie du théâtre et de celle de la culture en France en général.

La nôtre n'a pas pour vocation d'y répondre sinon de déposer quelques balises. Du reste, le paradoxe susdit est lui-même créateur d'antinomies ; présente dans l'Enclopcedia Universalis, l'écrivain génère, sur Internet, des centaines et de centaines de corrélats ; une bibliographie 
complète révélerait d'autres centaines d'articles, de la simple recension à l'étude plus fournie ou plus volumineuse. Il fut un temps où les grands quotidiens nationaux évoquaient les passages d'Atlan en Avignon, lorsqu'elle cohabitait en titre avec Edward Bond par exemple. Ce n'est pas le tarissement de l'inspiration qui explique, à nos yeux, les mesquineries de la presse, quand ce ne serait sa feinte indifférence. Il faudrait voir ailleurs : l'avarice intellectuelle ne serait pas la moins compromise. Les plus grands livres de l'écrivain interrogent sans discontinuer un mystère : la tuerie partielle de son peuple s'est déroulée dans la discrétion des nations et dans la surdité totale de Dieu, c'est-à-dire, à ses yeux, du Meilleur en l'homme. Soit. Aura-t-elle joué de malchance à l'heure des amalgames odieux et à la confusion totale des genres quand monte des confins une réclamation : la sainteté de la cause des peuples opprimés qui se voient tels et se pensent tels ? La mise en retrait de notre auteur ne saurait trouver dans cette allégation, qu'elle avance, une justification entièrement valable, mais a-t-on le droit de l'invalider ? Nous ne le croyons pas. Il reste qu'à l'œuvre humaniste d'Atlan on a préféré des ouvrages parfois émouvants mais de petite envergure : il leur manquera cette morale de l'Autre, cette esthétique du pittoresque sur la ligne tendue de l'Histoire convoquée, et de quelle sobriété, dans les jeux multiples d'une écriture qui sait prendre à Rabelais et à Albert Cohen une couleur et l'amalgamer à sa propre palette. On a toujours distingué les médiocres et laissé les inclassables se hisser à leurs dépens ou à leur péril.

PAR DÉFINITION, l'œuvre de Liliane Atlan échappe au processus de fabrication des étoiles filantes sur le firmament littéraire ; mais sa vigueur et son énergie auraient dû, depuis longtemps, interpeller les éditeurs réputés par leur catalogue, dans lequel tout écrivain digne du vocable souhaite figurer. Là aussi l'énigme qu'elle incarne continue de tourmenter l'observateur que heurtent des contradictions injustifiées. L'arbitraire, en matière de publication, ne répond pas tant à des critères économiques qu'à une vue du monde imposée par certaine élite. Un livre est un livre mais les conditions à son accessibilité sont souvent subordonnées à la relation de dépendance que certain système de diffusion subit; cette pression-là opère en amont et en aval une sélection qui n'est pas guidée par le bon goût, beaucoup s'en faudrait, mais qui détermine un jeu d'offre dont les mieux pourvus tireront les ficelles. Les éditeurs qui, contraints ou libres, travaillent différemment, aboutissent à des résultats assez extravagants : ils ne sont pas pris en compte, ou peu, dans le circuit des bons points. Le travail d'Atlan, coincé dans ces tenailles, est un peu au Bois dormant : si important soit-il, il n'a pas pu profiter de la relative souplesse d'intégration dans les modèles de recevabilité de son éditeur de substitution; et au lieu de s'irradier du prestige certain de cette écriture, l'éditeur a traité telle production en marchandise égale à telle thèse en économie ou en sociologie. Nous venons de voir comment une malchance technique, accouplée à une ambiguïté culturelle, a eu des effets funestes sur une création asphyxiée de l'extérieur. Tant de déveine est cas assez rare dans la vie d'un écrivain de cette pointure-là, et s'il n'est pas unique, il n'en est pas moins scandaleux.

MAIS SOUTENANCES, traductions, émerveillement préservent le feu sacré : comme si un aréopage de résistants continue d'ourdir une conjuration remarquable — sortilège d'une œuvre peu jouée, qui dispose d'une vitalité suffisante pour frapper les esprits, une communauté d'aficionados. Cette empathie ne s'engendre pas par miracle : elle tire son essence d'une réalité — la très grande 
force de l'œuvre, ni plus ni moins.

Clopin-clopant, l'Université, plus soucieuse du contenu que du paratexte, ne l'a pas boudée : il y a longtemps, reconnaissons-le, qu'elle en a repéré l'importance. Mais ce travail critique se joue dans le confinement eu égard aux conditions d'approche et de réalisation ; pour qu'il puisse disposer d'un impact évident, il faudrait que l'Université soit à même de créer une situation de prestige que sa crise profonde, et partout dans le vaste monde, décrédibilise : nous ne sommes plus au $19^{\mathrm{e}}$ siècle, voire dans la première moitié du $20^{\mathrm{e}}$ siècle, quand son rayonnement opérait sur toute la pyramide sociale ; la civilisation numérique en cours, sans dégager des perspectives très nettes, accentue le flou de la culture actuelle, si tant est que nous ayons déjà embarqué sur l'un de ses vaisseaux, ce dont je doute ; en sorte que la critique universitaire, pourtant outillée et distanciée, est celle du pauvre quand il s'agit de mesurer l'impact et la novation d'un auteur.

HORS D'ELLE ou dans sa proximité, deux essais méritent le détour. D’abord celui de la spécialiste du théâtre français, Bettina Knapp, paru naguère chez Rodopi.

Ensuite celui de Leonard Rosmarin. C'est lui qui a attiré notre attention.

Son beau titre, Liliane Atlan ou la quête de la forme divine, a l'avantage de placer le curseur très haut ; cet essai est, actuellement, le plus abondant qui ait été consacré à notre écrivain.

Rosmarin a su dégager la thématique en jeu : travail de contention, puisqu'il s'agit d'interroger des ruines - puisqu'il s'agit également de se refuser à toute chimère qui conjuguât les lendemains du monde hors l'expérience du Mal. «L'insatisfaction qu'Atlan a toujours ressentie est le catalyseur de son idéalisme comme de son art », écrit M. Rosmarin, qui ajoute : «L'auteure a eu l'immense courage de se remettre en question. Elle n'a jamais eu peur de prendre des risques. [...] Puisque le propre de l'être humain est d'évoluer au fil du temps, il est nécessaire que la quête de la forme divine se poursuive indéfiniment et qu'elle s'orchestre sur plusieurs registres différents » (p. 18). On lit, ailleurs, p. 36, que « dès qu'[Atlan] s'y installe, son judaïsme défaillant redevient le pôle magnétique de son existence ». Peut-être ne sommes-nous pas convaincus de l'ambition que prête l'essayiste à l'écrivain, quand il écrit « qu'elle est persuadée de pouvoir reconstruire un univers mal conçu, ressusciter un peuple à moitié exterminé et laisser une empreinte durable sur cette terre »; l'on voit, néanmoins, vers quelles cimes il entend mener le débat.

Ce n'est pas à l'angle de nos interrogations que M. Rosmarin a charpenté son ouvrage, mais son carrefour n'est pas loin du nôtre ; la réception d'une œuvre appartient précisément à son histoire et à l'histoire de la littérature. Nous devions d'y insister.

Saluons l'honnêteté, la sympathie de M. Rosmarin.

Elles sont les mâts de son entreprise.

Elle lui firent composer, jadis, ses essais sur Albert Cohen et Emmanuel Levinas : c'est dire à quelle hauteur il place la dramaturge, à quel rang il l'adjoint. La clarté de son style, la structure de son bâti ont su rendre présente l'épopée d'une écriture et de son projet : lire son livre c'est avoir en tête des titres passés à l'universel, sinon en fait, en tout cas en puissance.

L'CEUVRE de Liliane Atlan est appelée à durer, de quelque manière coderait-on cette durée : nombreux, parmi ses fanatiques, en sont convaincus; dernier classique des conteurs juifs de haut vol ou pont entre deux rives, celle qu'on a voulu annihiler et celle qui a réhabilité la tradition des 
pères réduits en cendres, qu'importe — il s'agit, il s'est agi et il s'agira de rendre à l'auteur du Rêve des animaux rongeurs ce qui lui appartient : une place éminentissime.

Liliane Atlan ou la quête de la forme divine, de Léonard Rosmarin, L'Harmattan et éditions du Gref, Paris et Toronto, 2004 